\title{
Aesthethic Crown Lengthening Surgery Guided By Cone-Beam Computed Tomography- A 6 Months Follow-Up Case
}

\author{
Dias R0 ${ }^{1 *}$, Bonzanini $\mathrm{NP}^{2}$, Suzuki FAR ${ }^{1}$, Camargo $\mathrm{QRJ}^{1}$, Yashiki BM${ }^{1}$ and Nascimento $\mathrm{FD}^{3}$ \\ ${ }^{1}$ Department of Periodontics, University of Mogi das Cruzes-UMC, Brazil \\ ${ }^{2}$ Departmentof Oral Radiology, University of Mogi das Cruzes-UMC, Brazil \\ ${ }^{3}$ University of Mogi das Cruzes-UMC, Brazil
}

*Corresponding author: Rafael de Oliveira Dias, Department of periodontics, University of Mogi das Cruzes- UMC, Rua Dr. Cândido Xavier de Almeida e Souza, 200- Sala 1S-15, Zip Code: 08780-911, Mogi das Cruzes, Brazil

Submission: 海 November 10, 2017; Published: 悳 January 04, 2018

\begin{abstract}
The excessive gingival display is anesthetic problem that can affect patient simile. This aesthetic unwelcome issue can be caused by altered passive eruption and a surgical treatment can be required. The diagnosis and treatment plan are performed in these cases by using a manual probe, the cementenamel junction is located, and the gingival excess evaluated. However, this procedure is complex, and an experienced examiner is required to perform it properly. Based on it, the cone-beam computed tomography (CBCT) technique became an interesting alternative approach. The aim of this study is present a 6 months follow-up case of aesthetic crown lengthening guided by CBCT. According to the case reported above, the cone-beam computed tomography wearing a plastic lip retractor is a useful and predictable tool in the diagnosis and planning of aesthetic crown lengthening surgery.
\end{abstract}

Keywords: Gummy smile; Cone beam computed tomography; Crown lengthening

Abbreviations: CBTC: Cone Beam Computed Tomography; CJE: Cement Enamel Junction; BC: Bone Crest; GM: Gingival Margin

\section{Introduction}

The excessive gingival display is an esthetic problem that can affect patient simile. This aesthetic unwelcome issue can be caused by altered passive eruption that is a clinical condition in which the gingival margin is positioned coronally on the anatomic crown due to disorders in the eruptive patterns of the dentogingival, resulting in short clinical crowns [1-3]. The diagnoses and treatment plan are usually performed by using manual probe. In these cases, the cement-enamel junction (CEJ) must be located and the gingival excess detected $[3,4]$. However, this procedure is complex, and an experienced examiner is necessary to perform it correctly. Based on these challenges, the CBCT became an efficient alternative, since the anatomical structures can be measured more precisely, and making the diagnosis and the surgical planning more predictable. In this technic the patients are submitted to a regular CBCT wearing plastic lip retractors in order to prevent interferences of the soft tissues, lips, and cheeks [5]. After this procedure, we can analyze the bone crest (BC), the gingival margin (GM), the CEJ, and the gingival excess more accurately. In addition, using the software we can measure de gingival margin excess in relation to the CEJ and predict the area needed to perform the osteotomy. However, the higher levels of radiation compared to a conventional dental radiography it is still a problem that should be taken into account [6] and a precise indication for it is use is required. The CTBC improves the final results, since it is possible to accurately measure the buccal and the palatal bone crest. On the other side, these measurements are not possible by using the conventional dental radiography due the root surface overlap. The aim of this case report is present a 6 months follow-up case of aesthetic crown lengthening guided by CBCT [7].

\section{Case Presentation}

First of all the patient was informed on the risks and benefits of the procedure and signed the informed consent. This case report was previously approved by the University of Mogi das Cruzes Ethics Committee in Clinical Research, under protocol 1.517.576. A 21 years old female patient presented at the Mogi das Cruzes University complaining about the excessive gingival display on the two laterals incisors (Figure $1 \& 2$ ). After then the CBCT scans were taken with an Orthophos XG 3D and the images (sections of $1.0 \mathrm{~mm}$ apart) were acquired using the software Galileos Viewer. The distances of the $\mathrm{BC}$ to the $\mathrm{CEJ}$ and the CEJ to the GM were evaluated (Figure 3). 


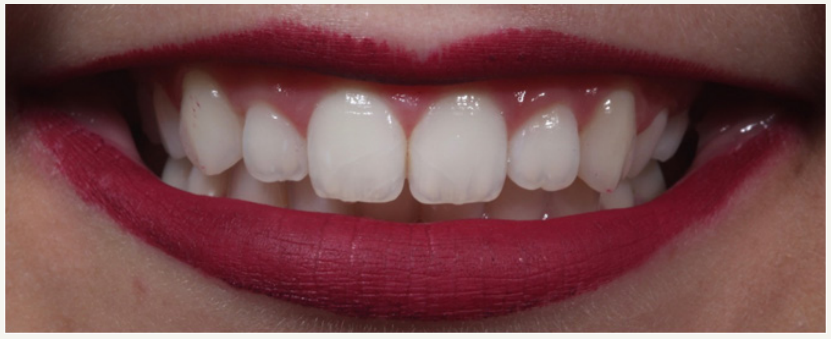

Figure 1: Lateral incisors aspect's preoperative.

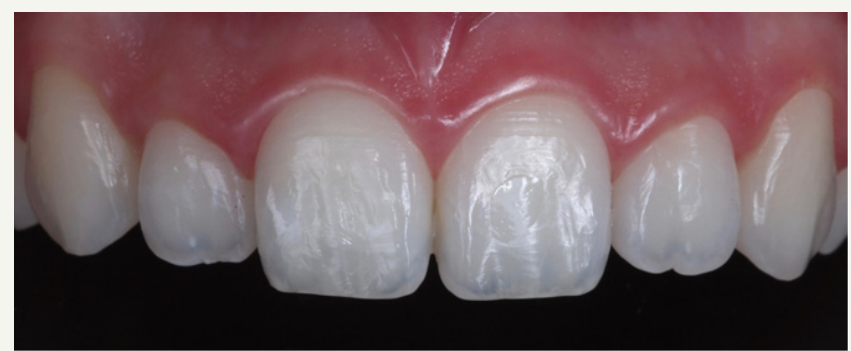

Figure 2: Lateral incisors aspect's preoperative.

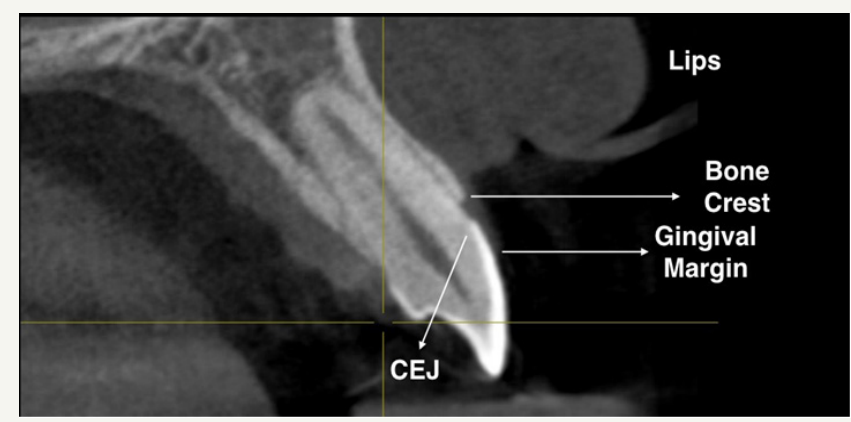

Figure 3: Image measurement using CBTC and patient wearing a plastic lip retractor.

\section{Procedure}

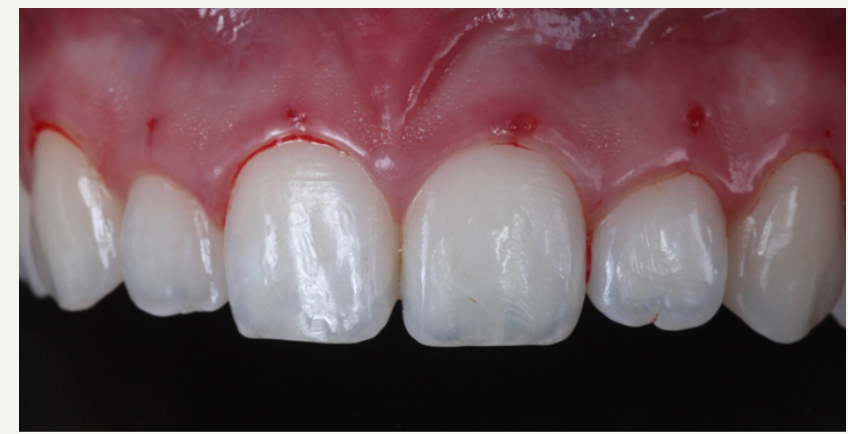

Figure 4: Bleeding points.

Under local anesthesia the measurements were transferred to the GM and bleeding points were performed to guide the gingival margin incisions in the teeth 13 to 23 (Figure 4). An internal bevel incisions were performed on these tissues followed by a sulcular incision (Figure 5) and then the gingival excess was removed (Figure 6).

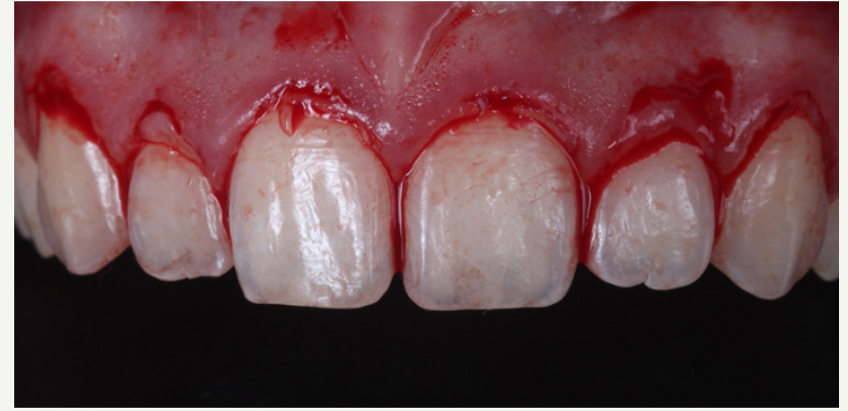

Figure 5: Internal beveled and sulcular incisions.

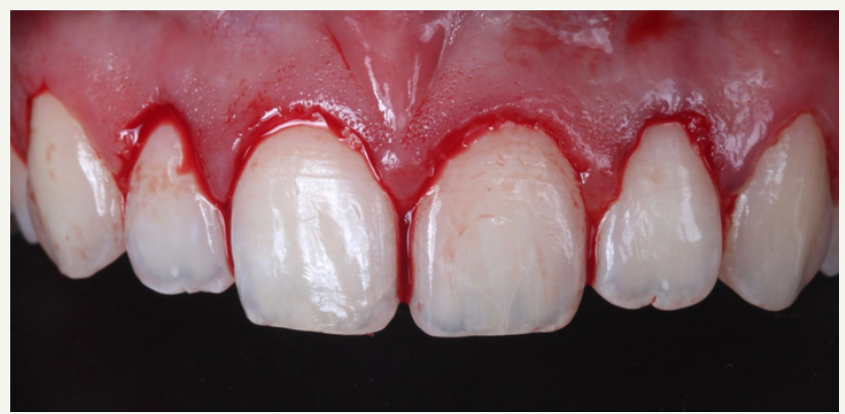

Figure 6: Gingival excess removal.

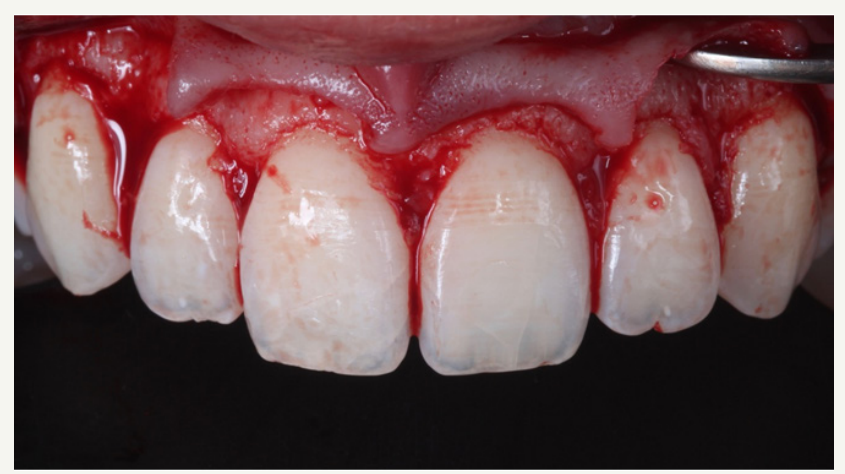

Figure 7: Full-thicknessmucoperiosteal flap.

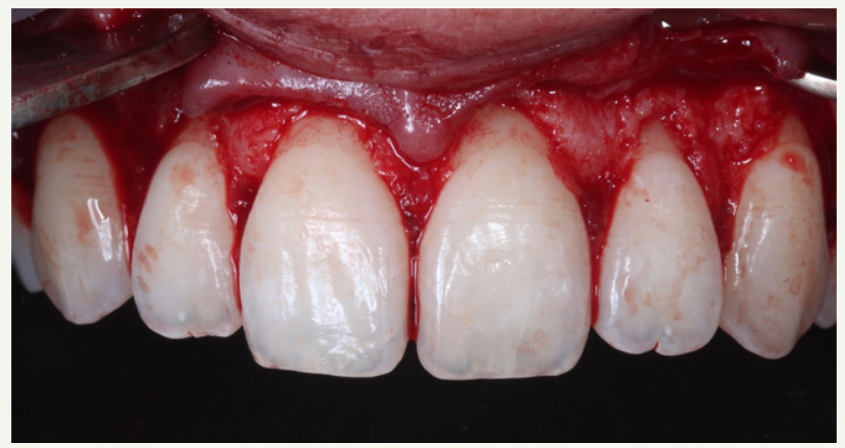

Figure 8: Osteotomy aspect.

A full-thickness mucoperiosteal flap was elevated to analyze the distance of the $\mathrm{BC}$ to $\mathrm{CEJ}$ (Figure 7), a $3 \mathrm{~mm}$ distance is necessary to preserve the biological width [7]. In this case report, the osteotomy 
was necessary, and it was performed by using a micro ochsenbein chisel (Figure 8). The flap was sutured on the interdental papilla with 5-0 nylon suture. The sutures were removed after 10 days (Figure 9). And them, the results were evaluated at 1,3 and 6 months after the surgical procedure (Figure 10-13).

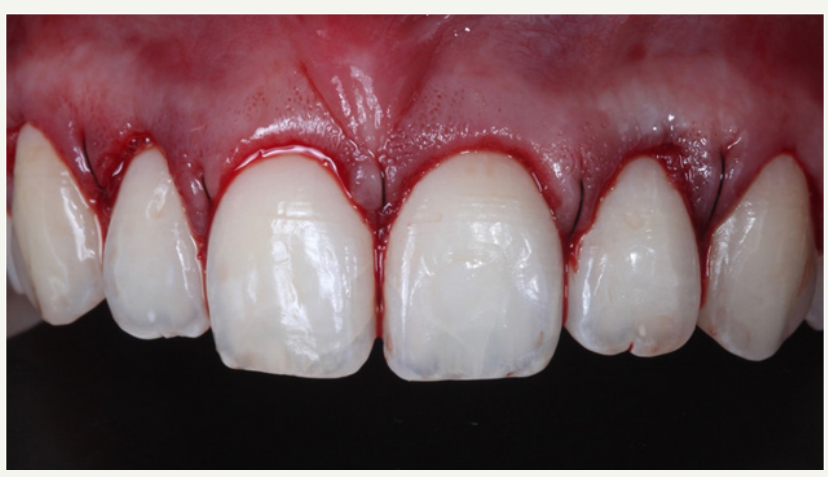

Figure 9: Immediate post operative.

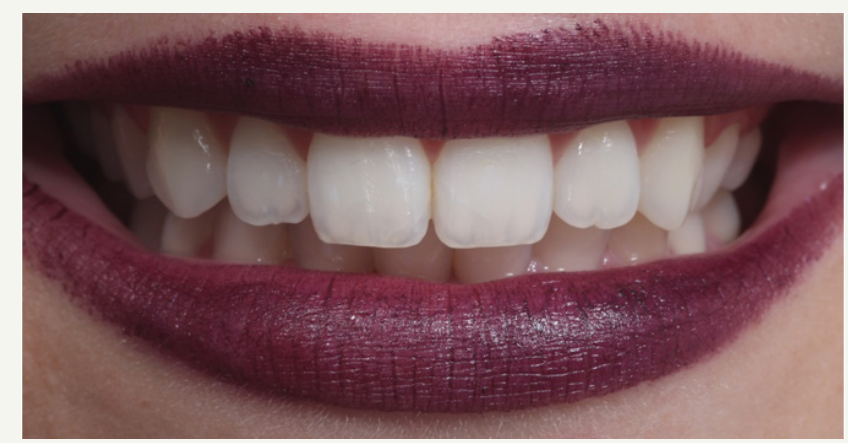

Figure 10: 3 months follow-up.

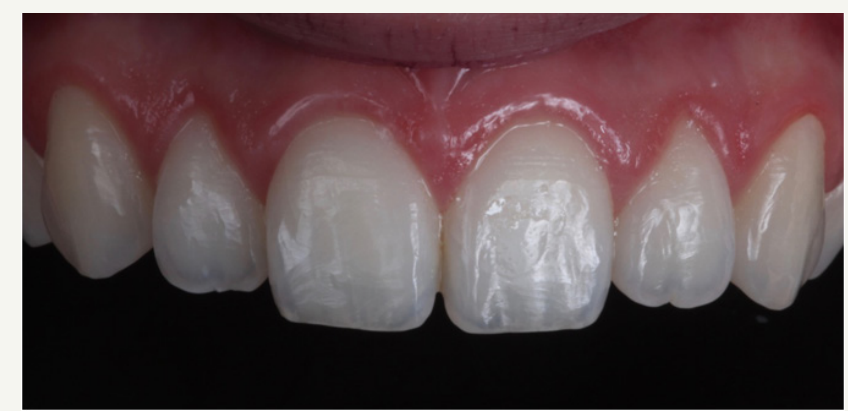

Figure 11: 3 months follow-up.

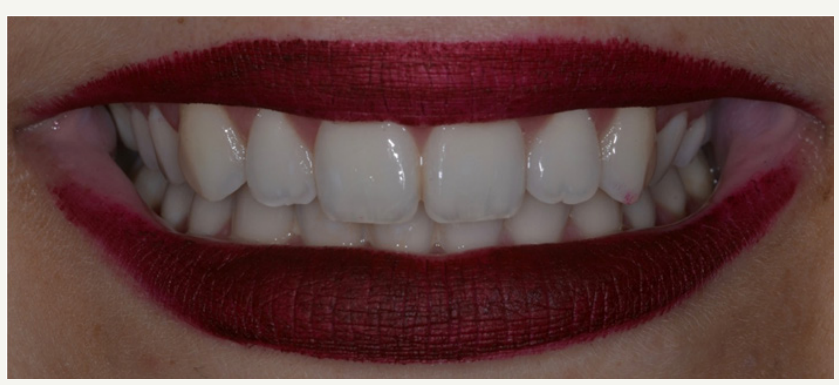

Figure 12: 6 months follow-up.

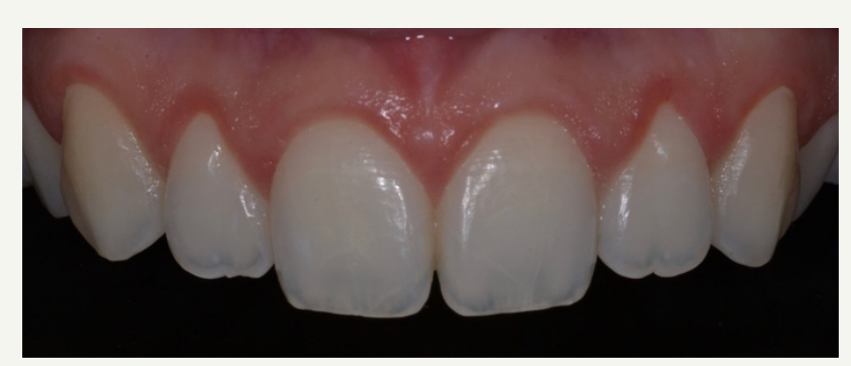

Figure 13: 6 months follow-up.

\section{Conclusion}

According to the case reported above, the CBCT wearing a plastic lip retractor is a useful and predictable tool in the diagnosis and planning of aesthetic crown lengthening surgery. Despite the excellent results obtained using CTBC, one cannot neglect the fact that the patient is submitted to a higher radiation rate when compared to the conventional dental radiography. Despite this and believing in an improvement in the quality of the surgical procedure the CTBC technique becomes a patient-centered alternative in aesthetic crown lengthening surgery.

\section{References}

1. Robbins JW (1999) Differential diagnosis and treatment of excess gingival display. Pract Periodontics Aesthet Dent 11(2): 265-272.

2. Levine RA, McGuire M (1997) The diagnosis and treatment of the gummy smile. Compend Contin Educ Dent 18(8): 757-762.

3. Fernanda FV, Hirata DY, Reis AF, Santos VR, Miranda TS, et al. (2014) Open-flap versus flapless esthetic crown lengthening: 12-month clinical outcomes of a randomized controlled clinical trial. J Periodontol 85(4): 536-544.

4. Camargo PM, Melnick PR, Camargo LM (2007) Clinical crown lengthening in the esthetic zone. J Calif Dent Assoc 35(7): 487-498.

5. Januário AL, Barriviera M, Duarte WR (2008) Soft tissue cone-beam computed tomography: a novel method for the measurement of gingival tissue and the dimensions of the dentogingival unit. J Esthet Restor Dent 20(6): 366-373.

6. Li G (2013) Patient radiation dose and protection from cone-beam computed tomography. Imaging Sci Dent 43(2): 63-69.

7. Gargiulo AW, Wentz FM, Orban B (1961) Dimensions and relations of the dentogingival junction in humans. J Periodontol 32(3): 261-267. 